\title{
Condition based maintenance using the cyber-physical system of industry revolution 4.0: A conception study
}

\author{
Othman Inayatullah ${ }^{1 *}$, Nor Asrina Ramlee ${ }^{2}$, Taharah Edin ${ }^{1}$ \\ ${ }^{1}$ Mechanical Engineering Programme, School of Engineering and Technology, University College of Technology Sarawak, Malaysia \\ ${ }^{2}$ Electrical Engineering Programme, School of Engineering and Technology, University College of Technology Sarawak, Malaysia
}

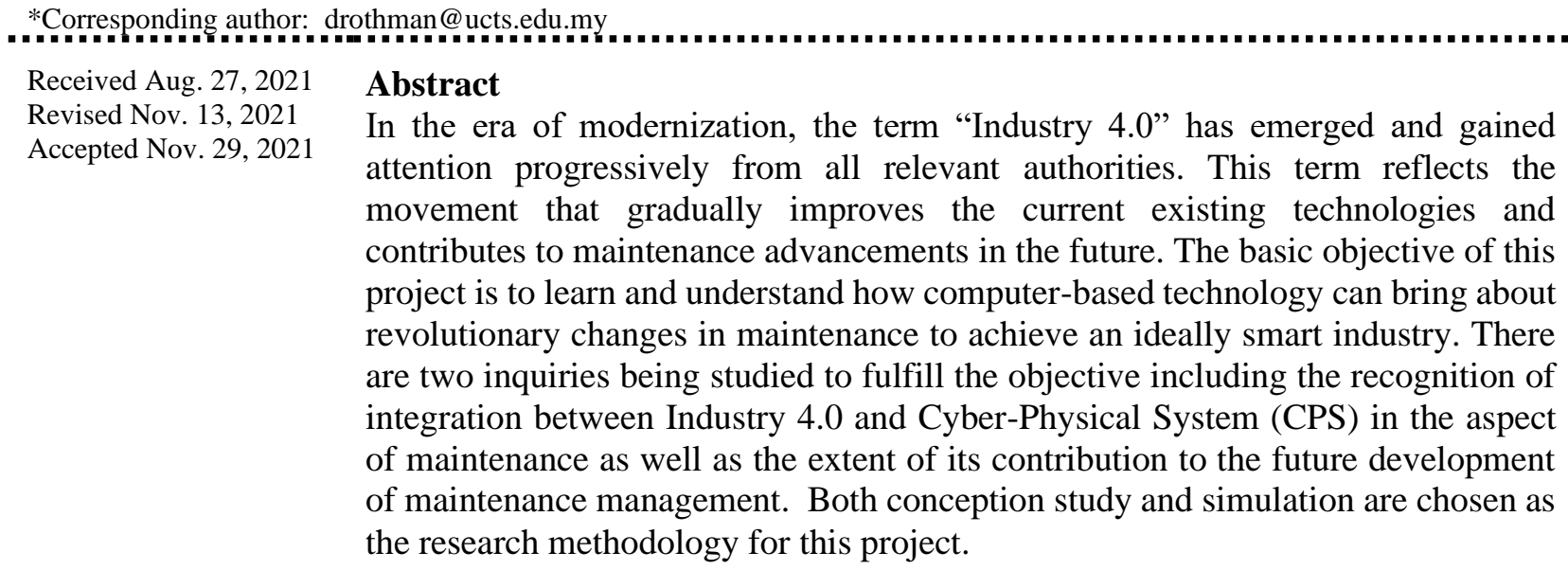

(C) The Author 2022.

Published by ARDA.

Keywords: Cyber-physical system; Maintenance; Industry revolution 4.0

\section{Introduction}

In a brief definition, the industrial revolution was the transformation to advanced manufacturing processes. Due to this advancement, local economies have shifted from traditional sectors like agricultural and handicraft economy to modern sectors like the industrial and mechanized economy. Most of the works and tasks, which were previously conducted manually, were currently being performed by motor-driven machines that significantly enhanced the working efficiency through mass production. This eventually results in easy, timesaving and commercially successful manufacturing processes.

In the era of modernization, the fourth industrial revolution (IR 4.0) began with the application of cyber-physical system (CPS). This allows various types of information to be carefully controlled and continuously connected between the physical industry level and the cyber-computational compartment. CPS is a platform which allows the collaboration between both the Internet of things and the networked services to fulfil IR 4.0. More specifically, CPS is described as breakthrough technologies which enable the control of linked systems constituted of physical resources and computer-based intelligence. CPSs allow both real and virtual worlds to come together to realize a closely linked world in which smart things cooperate with each other [1-6]. However, CPS is not the combination of both physical and computational worlds, instead, both of them intersect each other.

Besides, CPS has merged the engineering representation and mechanisms from a wide scope of relevant fields. During the utilization of CPS, a multipurpose core is provided to supervise, synchronize, command and unite the operations. This can be explained in such a way that CPS recognizes the physical world as input, convert input information into digital data, analyzes the data by computers and finally reacts back to the physical world as output. It is taken as a similar concept to the way how the internet affects the interaction between people [1$6]$.

This work is licensed under a Creative Commons Attribution License (https://creativecommons.org/licenses/by/4.0/) that allows others to share and adapt the material for any purpose (even commercially), in any medium with an acknowledgement of the work's authorship and initial publication in this journal. 
This concept is also applicable to CPS in which it redefines the human interacting and controlling manner towards the physical world. There are literally three fundamental components which are irreplaceable while considering a particular CPS. These components include cloud platforms, embedded systems and sensor networks. As for that, CPS is also delineated as a convergence of four different elements like embedded systems, real-time systems, distributed sensor systems and controls. In addition, CPS possesses some attributes including cross-domain sensor source and data transmission together with the embedded and mobile sensing.

Hence, the potential of CPS is driven by several new directions such as the expansion of cheap and enhanced sensors of progressively smaller size; the presence of cost-efficient, power-efficient, large storage space, smallsized computing equipment; the mobile revolution; large network bandwidth, and continual enhancements in energy-related fields [1-6]. In this project, CPS is emphasized for its adoption in condition-based maintenance as a holistic approach, where the concept of CPS in IR 4.0 is simulated in the temperature monitoring process.

\section{Adoption of cyber-physical system in maintenance}

In order to be adopted in typical manufacturing systems, cyber-physical systems (CPSs) should be made up of intelligent machines, storage structures and fabrication resources. These components of CPS are included with multiple purposes of freely interchanging data, responding and governing each other separately. However, there is no united design or common configuration of CPS that can be utilized widely for various applications due to the fact that such a system is still immature in terms of development.

Initially, CPS had been constituted of essentially two sections including the physical section and computing section. The physical section of CPS detects any stimulation in the physical dimension, gather information and perform the actions decided by the computing section, whereas the computing section interprets and organizes the data received from the physical section as well as for deciding on appropriate actions. As the CPS continued to make advancement, several unique structures or configurations had become apparent.

In this conception study, a basic model is utilized to demonstrate a particular cyber-physical system (CPS) with the aim to have a deep insight into the system. The common operating procedures of CPS can be classified into four critical steps such as monitoring, networking, computing and actuation. These steps are carried out in a sequential manner and repeated in a cycle. This operating cycle begins with the monitoring of physical processes and real-world condition which serves as a basic role of CPS.

It is also conducted with the purpose to provide feedback on previously taken actions by CPS and correct any faulty operations. It is then followed by networking which is responsible for accumulating and spreading data. After networking, CPS proceeds with computing in such a way that the system carries out the analytical process on the data gathered previously to verify the status of the physical process in achieving certain desired standards. Finally, actuation is taken place to perform decided actions concluded during the computation with the purpose to obtain the desired output.

In a short deduction, the CPS operating cycle demonstrates the data derivation process from sensors, the realworld data collection through an interlinked network, the justifiable calculated solution of the physical system conditions which could guide the controller to make proper instruction and control instructions being transmitted to the mover to carry out the responses respectively.

Through this structure, it becomes very obvious how the physical world is connected to the cyber world through digitalized information despite the fact that both of them are critically different. As followed by the operating structure, there are four substantial layers which include physical resource, industrial network, cloud, and supervision and control terminal.

As part of the physical resource layer, it is composed of many types of physical items like intelligent products, intelligent machines and smart conveyors. The industrial layer contributes to a significant foundation which allows an internal interaction between the physical items and also bridges the gap between the physical resource 
layer and the cloud layer. Cloud layer is basically a meshwork of servers which delivers the filmed servicing pattern of servicing infrastructure and servicing platform.

Lastly, the monitoring, analyzing and controlling of the terminal layer enables humans to acquire computed statistics from the cloud, utilize distinctive designs or conduct maintenance and diagnosis indirectly through the use of the Internet. As an overall result, the adoption of CPS in the maintenance systems creates a smart factory which takes an important role in the IR 4.0 projects [1].

\section{Conception study}

It is within an awareness that the Next Generation Maintenance solution brings a digital transformation to the service phase, which completely includes the end-to-end digital integration. With regard to engineering fundamentals, digital integration is the holistic development of digital engineering that covers aspects of product design and development. It involves the beginning of the production process which is production planning and production engineering before it enters the production and service phase. An important element in the service phase is engineering and maintenance management. In digital integration, all employees directly or indirectly involved in production have the ability to access real-time information and data. In addition, information and data controls must be comprehensive and cover all levels of personnel, in the areas of management, administration, finance, operations, violation and up to subordinate staff.

This is an important aspect of revolutionizing the manufacture and service of digital integration. In general, the IR 4.0 created a horizontal network at the strategic level and provided end-to-end vertical integration across the value chain at the business process level, including engineering, vertical integration capabilities and design of manufacturing network system. This is the basis for the integration of engineering processes IR 4.0.

\subsection{Temperature monitoring in maintenance system}

Fire and explosion have been considered the most frequent disastrous accidents in most factories as reviewed from the folds of history. The reason behind this is that the periodic monitoring in the preventive maintenance system is insufficient to pre-detect the overheating phenomenon of most mechanical systems or machine components. This explains why predictive maintenance strategies are essential especially when dealing with the temperature or pressure monitoring in assistance with CPS. In this case study, the accuracy of the temperature sensors and the self-interaction of the temperature monitoring system with CPS will be demonstrated through a simple simulation as to depict the effectiveness of CPS to achieve the prediction purpose and to detect any abnormal temperature rising before any fire breakouts.

\subsection{Simulations}

In order to simplify this case study of temperature monitoring, an electric kettle is used to simulate a boiler in real life and meanwhile, the electric kettle is installed with 4 thermal sensors on different parts of the kettle. For the overall computerized maintenance management systems (CMMS) simulation, an Arduino Uno R3 development board installed with an Arduino Wi-Fi router are used to study how the sensors interact with the system and alert the users or operators automatically via the intranet or internet. The simulation procedures are as follows:

a. All the necessary materials and equipment are prepared as follows,

Table 1. Material list for fabrication of the simulation apparatus

\begin{tabular}{lc}
\hline Material/Equipment & Quantity \\
\hline Electric Kettle & 1 \\
Arduino Uno R3 Processor & 1 \\
Circuit Board & 1 \\
Arduino WiFi Router & 1 \\
Arduino Router Adapter & 1 \\
Thermo Sensor & 4 \\
USB Connector & 1 \\
Soldering Device & 1 set \\
Connecting Wire & $1 \mathrm{~m}$ \\
Power Bank & 1 \\
Sellotape & $1 \mathrm{ring}$ \\
\hline
\end{tabular}


b. Arduino Uno R3 processor, Arduino Wi-Fi router and adapter are soldered on the circuit board and connected with each other. Four thermal sensors are connected to the processor by plugging them onto the pins as shown in Figure 1.

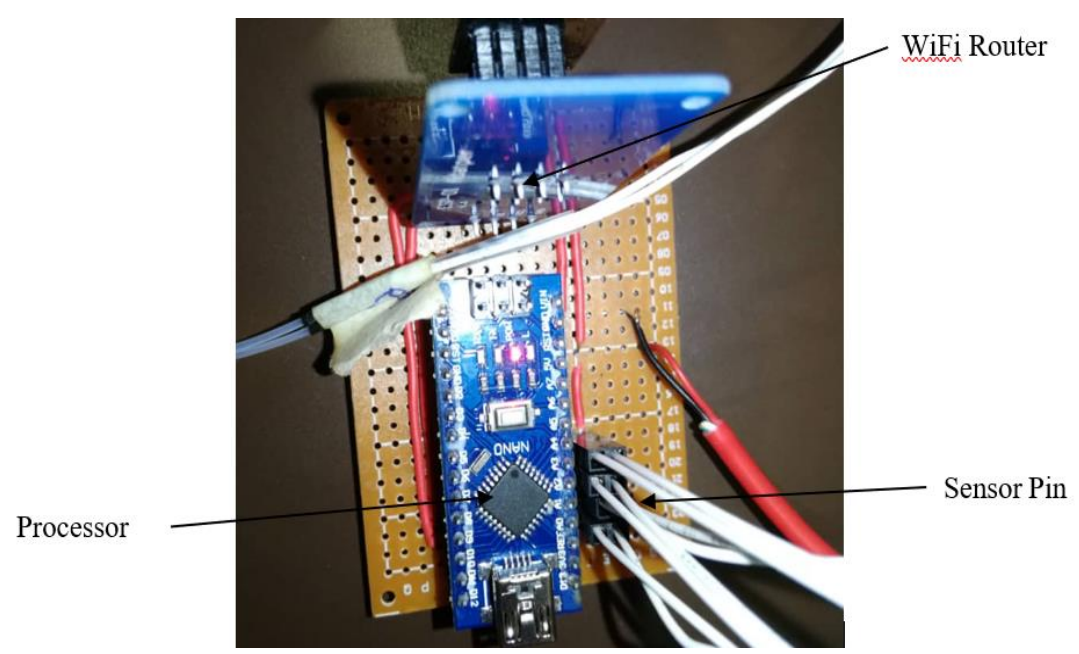

Figure 1. Installation of processor, Wi-Fi router, and sensors on circuit board

c. Figure 2 showing the thermal sensor probes attached to different locations of the electric kettle with tape.

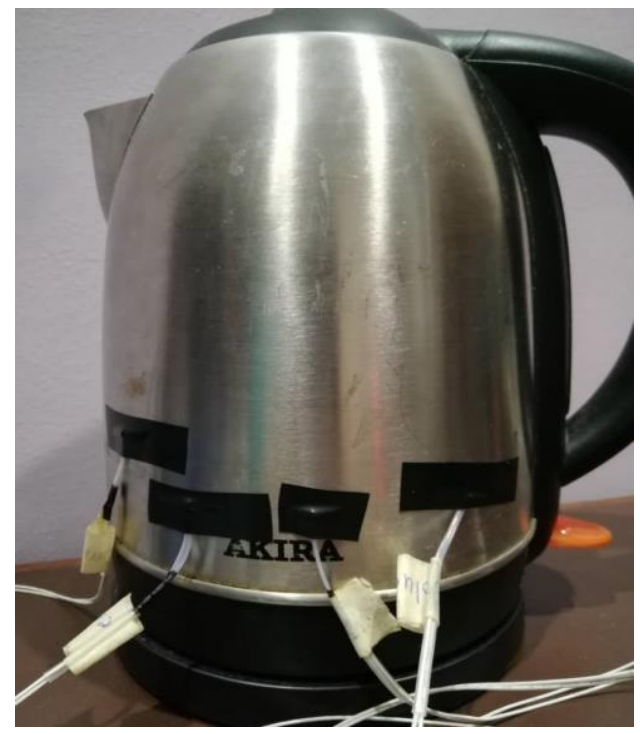

Figure 2. Attaching thermal sensors on an electric kettle

d. Figure 3 showing the circuit board then connected to the computer for the installation of a temperature monitoring program. After that, the circuit is reconnected to a power bank as its power source.

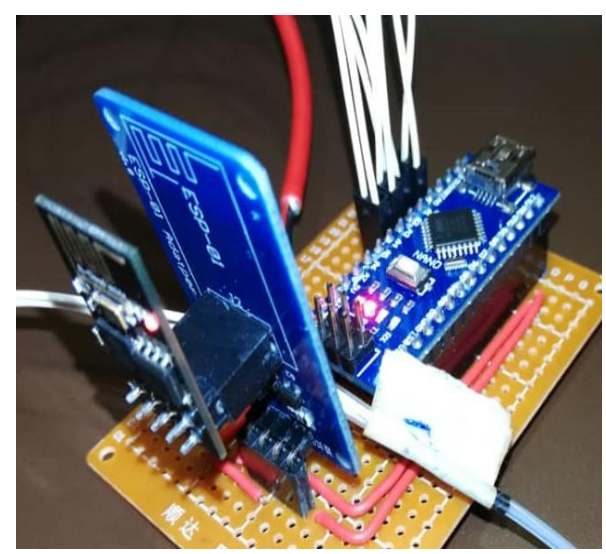

Figure 3. Connection of circuit board to a power source 
e. The temperature monitoring software is run simultaneously on the laptop and any electronic gadget such as a tablet which is connected to the same Wi-Fi as shown in Figure 4. Initial temperatures of the electric kettle from different sensors are read and checked on both laptop and tablet to ensure $100 \%$ collaboration.

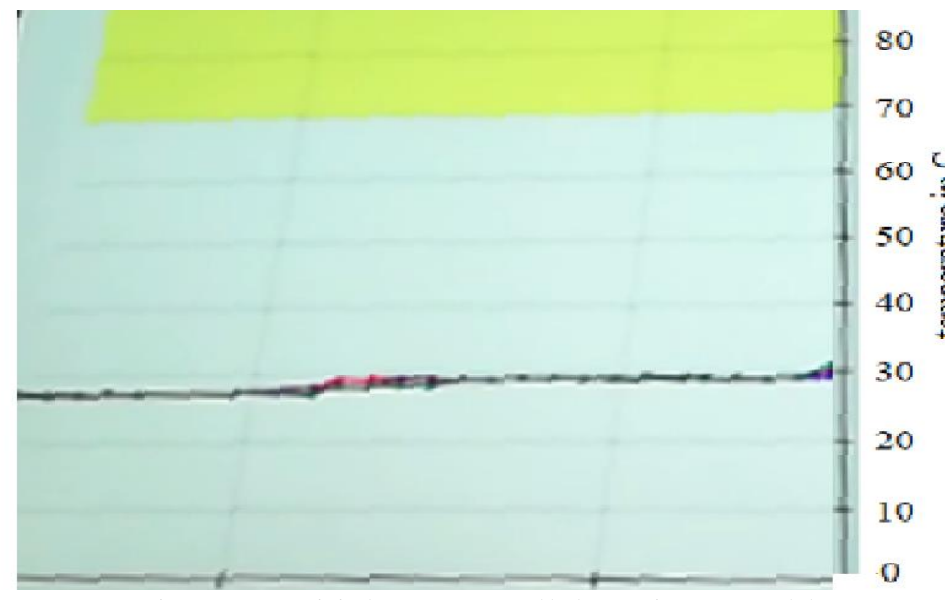

Figure 4. Initial system collaboration on tablet

f. After the system set-up is completed, the experiment is started by filling the electric kettle with water. The electric kettle is connected to a power socket and then switched on to boil the water. During the boiling process, the rise and variation of temperature at different locations are depicted on a graph. Each temperature trendline read from 4 thermal sensors is represented by different colours as shown in Figure 5.

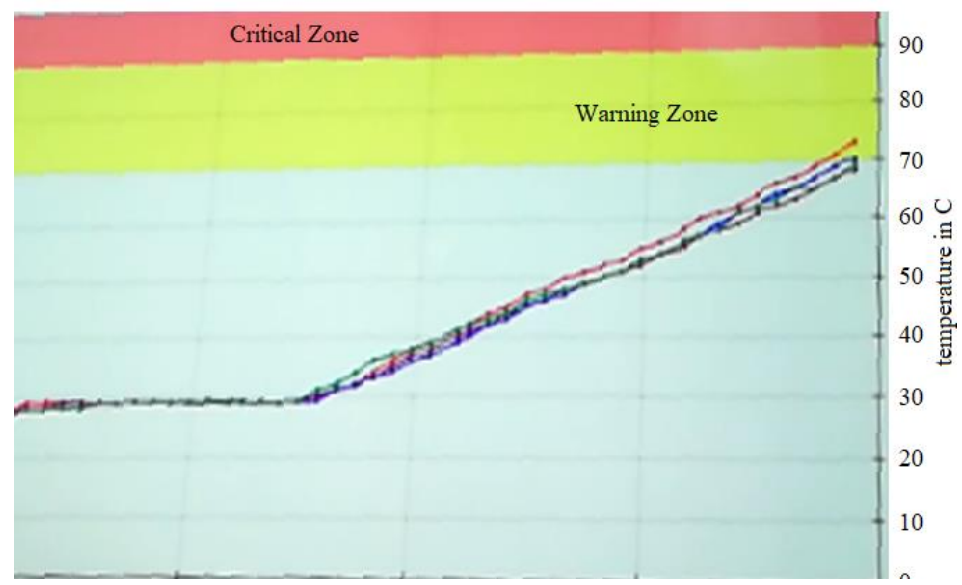

Figure 5. Rising of temperature during boiling

g. Once the temperature of any sensor reaches $70^{\circ} \mathrm{C}$, a notification email will be sent to the operator and posed as an alert on the tablet screen as shown in Figure 6.

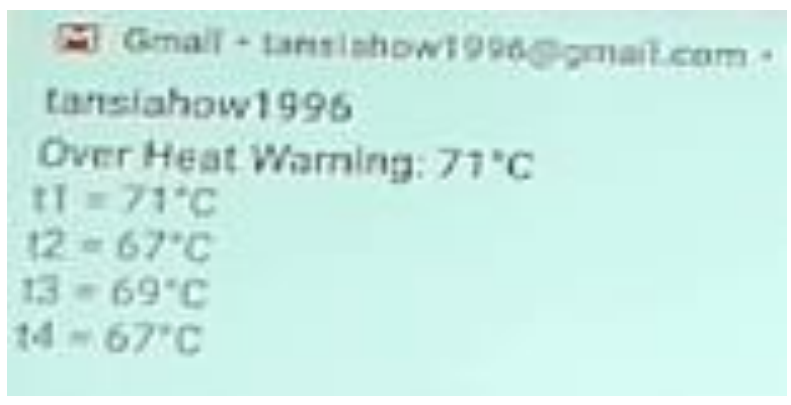

Figure 6. Warning notification pop out on tablet screen 
h. When the boiling temperature reaches $90^{\circ} \mathrm{C}$, the system will send another notification email to the operator to inform that the temperature has reached a critical zone and the boiling is nearly complete.

\section{Results and discussion}

Based on the simulation had performed before, the effectiveness of the system is very high as the notification or alert email was shown on-screen within seconds after the temperature reaches the warning zone and critical zone. Looking at the case of an industrial boiler or any mechanical component, the warning zone serves as the first predictive strategy. Once the temperature reaches the warning zone, this means that the machine has encountered abnormal temperature rise and hence predictive maintenance is achieved. The second-stage warning belongs to the critical level. This means that the identified part of the machine is overheated critically and it needs mandatory breakdown maintenance. Otherwise, a fire or explosion may break out consequently. By this, it has successfully simulated the working process of CPS in the maintenance system of IR 4.0 which condition-based maintenance is achieved in a predictive maintenance system. This is essential because the predictive maintenance strategy in IR 4.0 with the help of CPS is able to predict failure before any disastrous circumstances and loss happen.

\section{Conclusion}

Overall, this project has successfully demonstrated the integration between two distinctive terms of IR and CPS in the aspect of condition-based maintenance and maintenance system management. It also shows the potential of such a combination for enhancing the current industry environment in the maintenance perspective. It can be deduced that the arising technologies generated through this integration provide fresh insights to initiate alternative maintenance strategies and techniques in a more advanced manner. In terms of the condition-based maintenance and maintenance system management, CPS functions to collect exact realistic data of the production system with the utilization of smart sensors and then it systemizes, classifies, also submits the information to the user. Under IR 4.0, the technologies involved are capable of being self-aware, self-predicting and self-reconfiguring through the application of the smart data analytics and smart devices. Through the combination of IR 4.0 and CPS, decentralization can be realized in which the smart devices can gain access and interpret massive information by themselves and provide responses automatically. In this way, there is a high possibility that a fully automated production and maintenance system which operates continuously and optimally can be achieved even with the absence of manpower.

\section{Acknowledgements}

This work was supported by the research grant (UCTS/RESEARCH/4/2019/13-01) of University College of Technology Sarawak.

\section{Declaration of competing interest}

The authors declare that they have no any known financial or non-financial competing interests in any material discussed in this paper.

\section{References}

[1] K. He \& M. Jin, "Cyber-Physical System for maintenance in industry 4.0," Production Systems, pp. 6$22,2016$.

[2] C. Yu, X. Xu \& Y. Lu, "Computer-Integrated Manufacturing, Cyber-Physical Systems and Cloud Manufacturing - Concepts and relationship," Manufacturing Letters 6, pp. 5-9, 2015.

[3] R. Velmurugan and T. Dhingra, "Maintenance strategy selection and its impact in maintenance function," International Journal of Operations \& Production Management, vol. 35, no. 12, pp. 1622-1661, 2015.

[4] M. Brettel, N. Friederichsen, M. Keller and M. Rosenberg, "How Virtualization, Decentralization and Network Building Change the Manufacturing Landscape: An Industry 4.0 Perspective," International 
Journal of Mechanical, Aerospace, Industrial, Mechatronic and Manufacturing Engineering, vol. 8, no. 1, pp. 37-44, 2014.

[5] J. Lee, H.-A. Kao and S. Yang, "Service Innovation and Smart Analytics for Industry 4.0 and Big Data Environment," Procedia CIRP, no. 16, pp. 3-8, 2014.

[6] J. Lee, B. Bagheri and H.-A. Kao, "Recent Advances and Trends of Cyber-Physical Systems and Big Data Analytics in Industrial Informatics," In International Conference on Industrial Informatics (INDIN), 2014. 